\title{
NANO SENSORS FOR GAS DETECTION IN SPACE AND GROUND SUPPORT
} APPLICATIONS

\section{ABSTRACT}

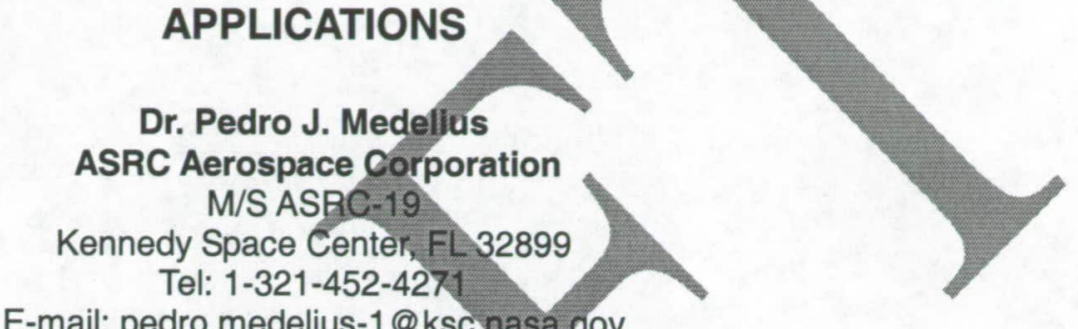

E-mail: pedro.medelius-1@ksc.nasa.gov

\section{CANEUS-11066}

Personnel living in a space environment as well as technicians and engineers preparing spacecraft for launch can potentially be exposed to small amounts of hazardous gases. It is therefore important to be able to detect, identify, and quantify the presence of a gas, especially when its presence could lead to a fatal situation.

The use of small and sensitive sensors can allow for the placement of these devices over a large area, thus allowing for a more precise and timely determination of a gas leak. ASRC Aerospace and its research partners are developing nano sensors for the detection of various gases, including but not limited to: $\mathrm{H}_{2}, \mathrm{NH}_{3}, \mathrm{~N}_{2} \mathrm{O}_{4}$, hydrazine, and others. Initial laboratory testing has demonstrated the capability to detect the gases in concentrations lower than parts per million. Testing and development is continuing to improve the response and recovery times, and to increase the sensitivity of the devices. Different coatings and electrodes are currently being evaluated to determine the optimum configuration for the detection of a variety of gases.

The small footprint of the nano sensors allows for several devices, each responsive in a different way to different gases, to be placed into a single substrate. Multiple devices embedded into a single substrate results in increased reliability and in a decrease in the need for periodic calibrations. The use of different coatings will result in a small electronic nose capable of distinguishing between different gases. A multi-channel signal conditioner amplifier built on a small multi chip module is used to process the output of the sensors and to deliver a signal that can be remotely monitored. All the data is digitized and transmitted over the same cable pair used to power the amplifier. Multiple outputs can be connected to a single cable pair in order to minimize the added weight and expense associated with cabling in a spacecraft.

The sensors will be run through a qualification process to evaluate their suitability for space applications. We are expecting to have fully functional sensors available for initial field deployment and testing by the end of the year 2006.

ASRC Aerospace is the prime contractor at the Kennedy Space Center for the University Affiliated Technology Development Contract (USTDC).

\section{INTRODUCTION}

Gas sensors are indispensable to monitor the quality of the environment in areas where hazardous gases might be present. A typical launch environment involves the use of $\mathrm{H}_{2}, \mathrm{NH}_{3}$, $\mathrm{N}_{2} \mathrm{O}_{4}, \mathrm{~N}_{2}, \mathrm{He}$, hydrazine, and others.

The Space Shuttle uses liquid hydrogen and liquid oxygen as the fuel and oxidizer, respectively, to power its three main engines. Because of the potential hazards associated with these gases, it is important to ensure that no gas leaks are present. NASA also monitors areas within the Space Shuttle for helium (an area purge gas), and argon (a marker for ambient air leaks) in a nitrogen background. From the beginning of the Space Shuttle program, mass spectrometers have been used to monitor for cryogenic fuel leaks. Mass spectrometry is a proven technology that can be used to monitor all necessary gas 
constituents at the required limits of detection [low part per million (ppm)]. However, the size of the mass spectrometers is such that all the monitoring is done on the ground, and not in the vehicle itself. Figure 1 shows a mass spectrometer rack in use at the Space Shuttle launch pads. One of the drawbacks of remotely sensing for gases is that gas samples have to be transported to the mass spectrometer through long transport lines (about $115 \mathrm{~m}$ long) because the size of the Space Shuttle Orbiter. This means the samples being analyzed are tens of seconds old by the time they reach the mass spectrometer. Figure 2 depicts the Space Shuttle Orbiter on top of the Mobile Launch Platform. The mass spectrometer is located a few levels below the pad platform.
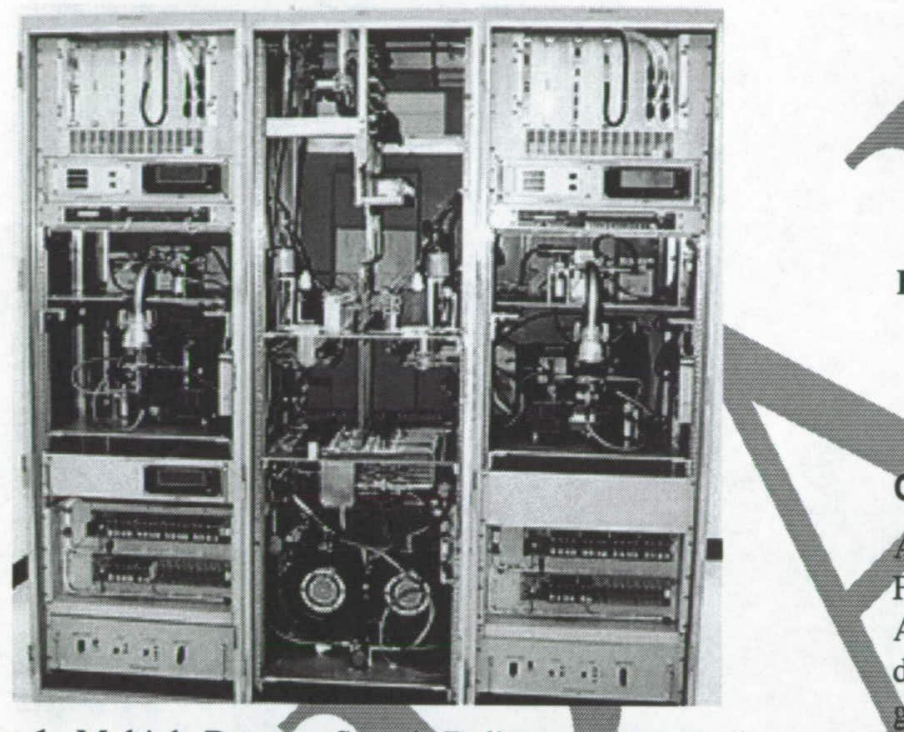

Figure 1. Multiple Detector Sample Delivery System used to deliver gas samples to the inlets of twa independent mass spectrometers.

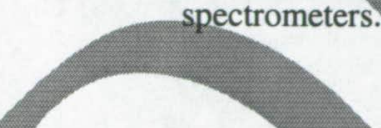

The use of small sensors, with sensitivity and selectivity similar to that achieved by the mass spectrometer, can result in a more timely analysis of the gas samples, especially when such sensors are physically placed in the area of interest. The main challenges are to be able to design sensors with fast response and recovery times, and to selectively design the sensors to respond to different gases.

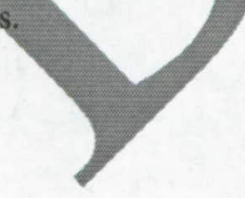

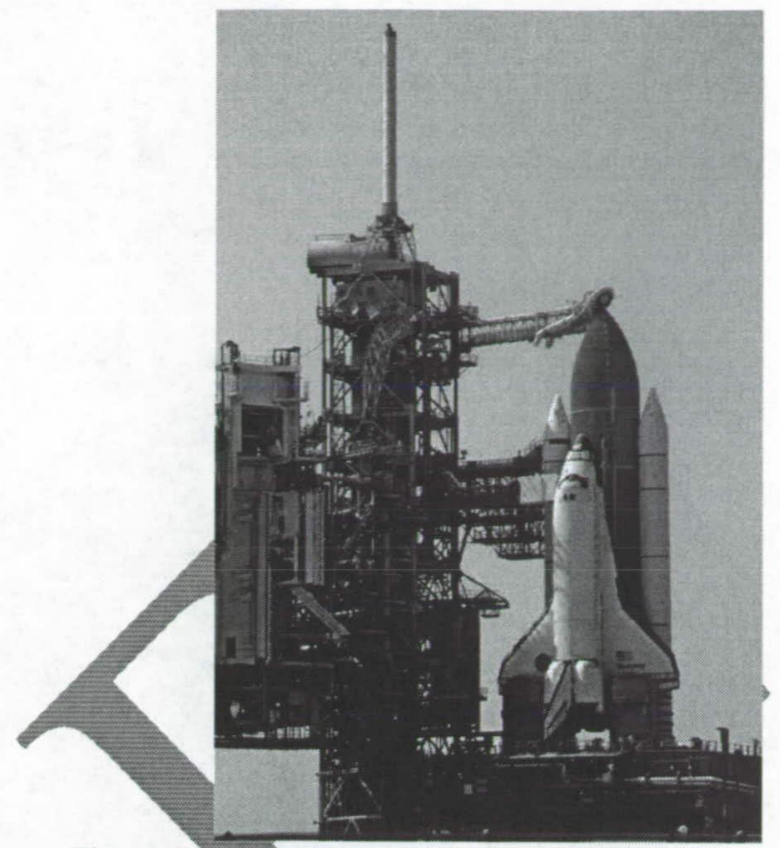

Figure 2. Space Shuttle Discovery in full launch configuration. The gaseous oxygen vent arm has been extended to the top of the External Tank.

\section{CURRENT EFFORTS}

ASRC Aerospace and its partners: the University of Central Florida, the Center for Nanotechnology Research at NASA Ames Research Center, and NASA Glenn Research Center are developing nano sensors for the detection of various types of gases, Applied research in nanotechnology is vital to the development of future space vehicles, as it will enable materials and devices with reduced weight, cost and power requirements. All these factors are important requirements for long duration space travel.

ASRC Aerospace Corporation and the Center for Nanotechnology at NASA Ames Research Center have reached an agreement to jointly develop, manufacture, and test nano devices. The Center for Nanotechnology has the best infrastructure and resources to perform low Technology Readiness Level (TRL) research in nanotechnology, while ASRC is in the best position to apply the Center of Nanotechnology's research in launch site ground support and space applications, through prototyping, testing and evaluation.

An ASRC researcher located at NASA Ames Center for Nanotechnology is currently performing the following activities:

- Conducting experiments on nano materials.

- Developing, testing and evaluating nano sensors and other nanotechnology based devices. 
- Investigating new technologies leading to more sensitive and more accurate sensors.

- Identifying applications and requirements for nano sensors for ground support and space applications.

The initial effort has concentrated on developing a sensor and optimizing its response to gaseous $\mathrm{N}_{2} \mathrm{O}_{4}$ and $\mathrm{NO}_{2}$. Different coatings continue to be tested so the optimum configuration can be selected.

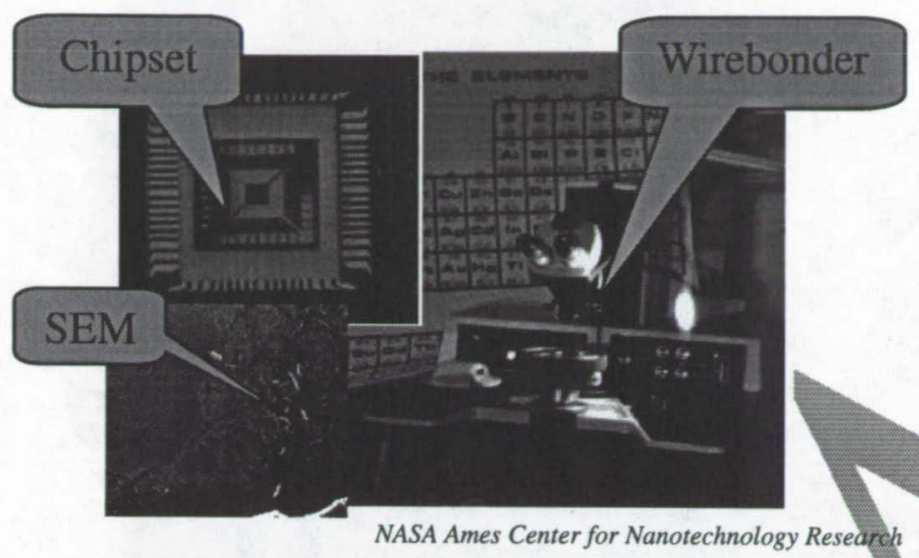

Figure 3. Wire bond station for the multi-IDE sensors chip developed for $\mathrm{NO}_{2}$ detection

Initial testing of the sensors was conducted for various gaseous $\mathrm{NO}_{2}$ concentrations and different relative humidity conditions, with some initial results shown in Figure 4. Several coatings have be tested to date, with IDE gap sizes of $8,12,25$, and 50-mm.

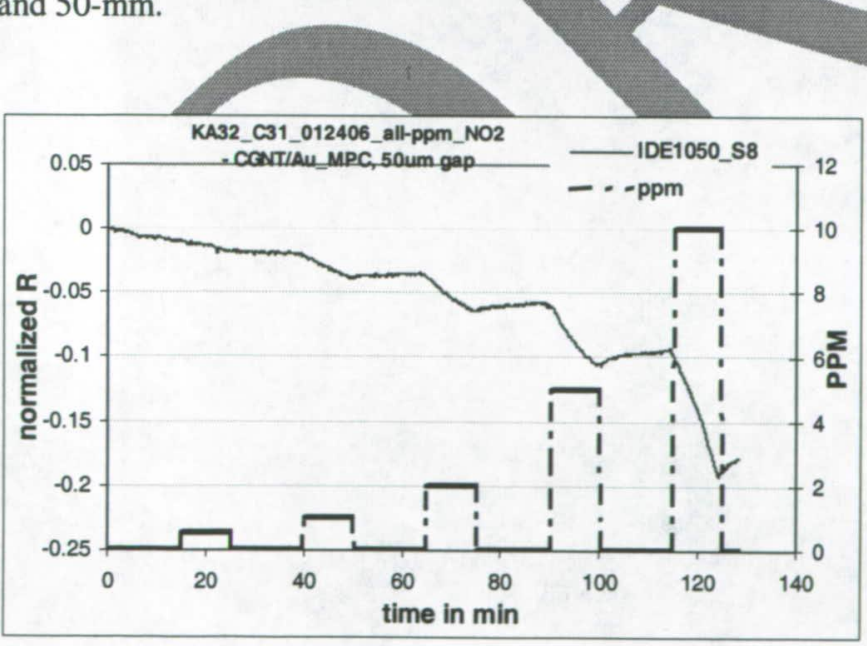

NASA Ames Center for Nanotechnology Research

Figure 4. Changes in normalized resistance in the presence of various gas concentrations.

One of the immediate objectives is to optimize the dimensions of the IDE gap sizes and the coatings on the electrodes so that the sensitivity to the gas is maximized. Two important elements to assess the usefulness of the sensor are the detection and recovery times. Optimization of these factors is in progress

The test setup used to characterize the response of the sensors is presented in Figure 5, and the testing profile for the injection of the gases is shown in Figure 6. The environment if purged with air for a certain time, typically for a duration of 5 to 10 minutes. Following the purge cycle, the gas is applied to the sensor at a preseribed concentration. The resistance between the electrodes is then monitored and recorded in real time. Multiple sensors can be tested simultaneously, thus allowing for direct comparison among different nano material coatings and electrode gaps.

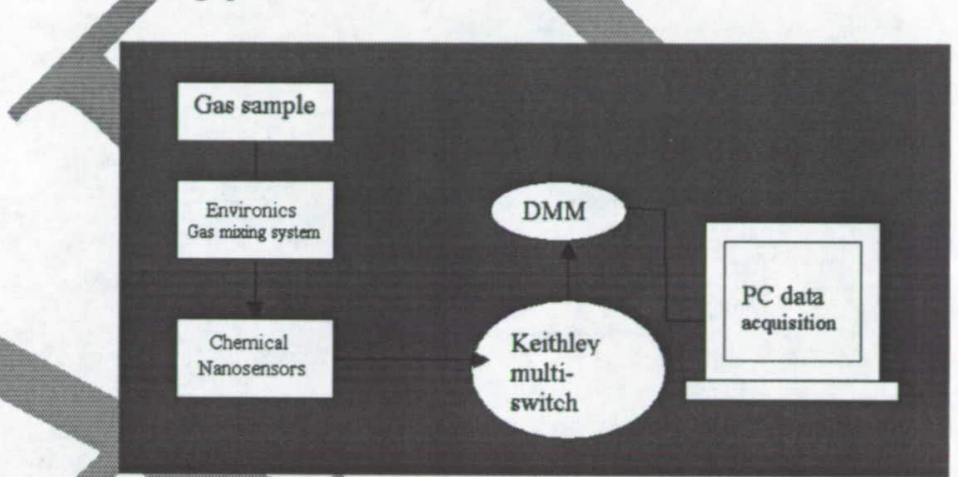

NASA Ames Center for Nanotechnology Research Figure 5. Typical experimental setup

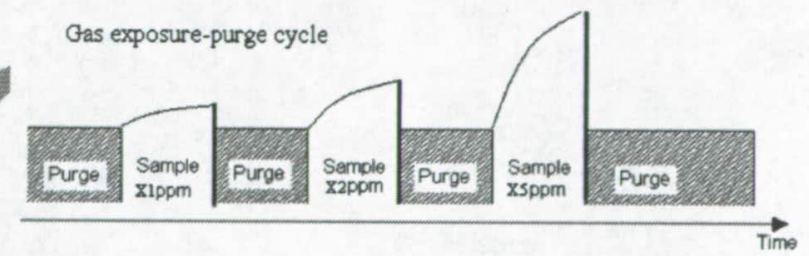

Figure 6. Exposure-purge steps of the chemical sensing

Although the initial work has concentrated on the detection of $\mathrm{NO}_{2}$ and $\mathrm{N}_{2} \mathrm{O}_{4}$, the effort is not limited to those gases. Following the completion of the initial development and testing of these sensors with the aforementioned gases, testing will start with $\mathrm{H}_{2}$ and $\mathrm{NH}_{3}$. The final objective will be to optimize individual responses to various gases, and to integrate the sensors onto a single substrate. Multiple sensors in a single device will result in increased reliability and selectivity.

Collaborative work with the University of Central Florida is centered on the development of nano sensors for the detection of gaseous hydrogen. The overall goal of this project is to develop low or room temperature based hydrogen $\left(\mathrm{H}_{2}\right)$ sensors that can be used for NASA's space program. Of particular emphasis are the cheap, robust, highly responsive, selective 
(one of the major issues discussed in the National Nanotechnology Initiative (NNI) 2004 Conference- NASA Grand Challenge Workshop-Sensor Session-Aug 04), fast operating, low temperature (room and cryogenic) sensors detecting $\mathrm{H}_{2}$ at ppm level. The advanced concepts in $\mathrm{H}_{2}$ separation and gas sensing technologies are merged, for the first time, for the development of a novel $\mathrm{H}_{2}$ sensor consisting of porous, doped, nano-clustered particulate/fiber/rod shaped nanostructures.

Furthermore, the sensor will also be a unique integration of nanomaterials and MEMS devices, thus combining the expertise from materials, nanoscience, and MEMS fabrication technology. While selectivity and sensitivity are key challenges for $\mathrm{H}_{2}$ sensing at room temperature, reduction in the particle size and shape and their integration in a MEMS architecture is sought to overcome these challenges.

Figure 7 shows a MEMS device which has been successfully developed and coated with the sensor-material via sol-gel dipcoating technique at the University of Central Florida.

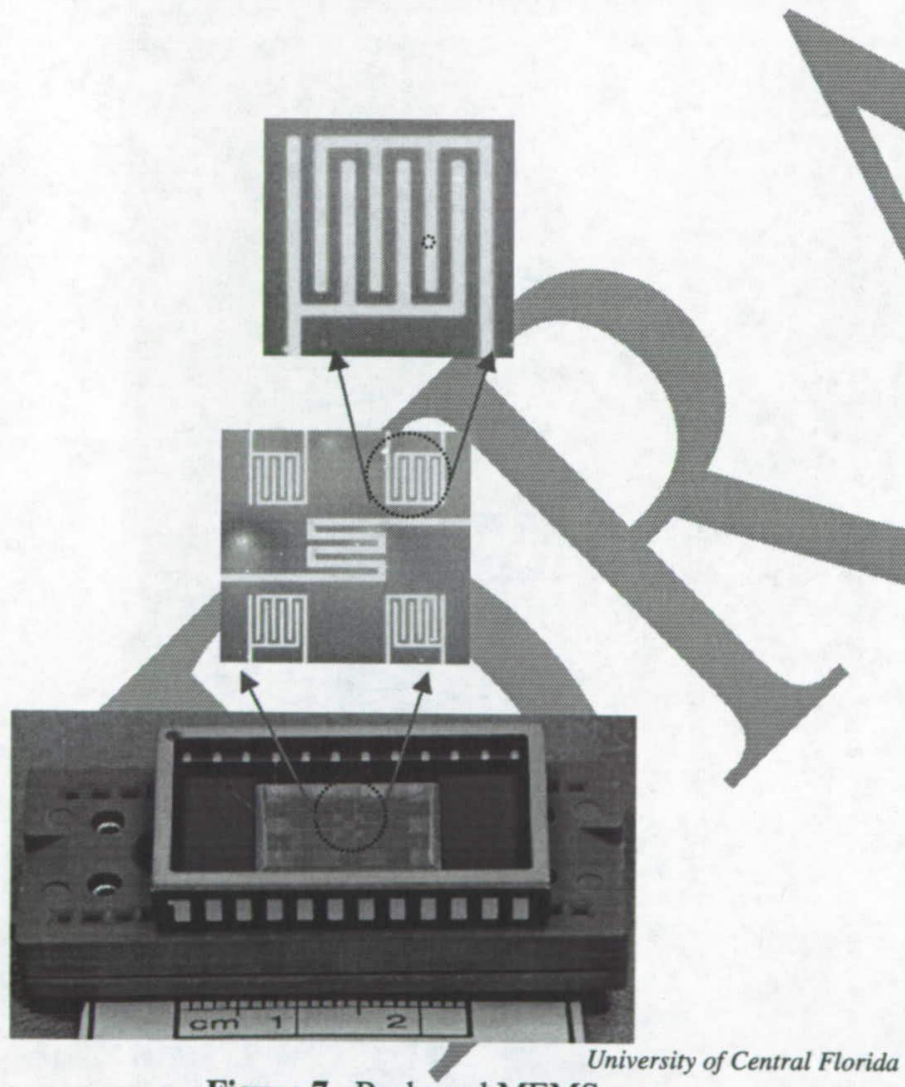

Figure 7. Packaged MEMS sensor.
For initial testing purposes, the nano wires were grown on silicon substrate directly bonded to a ceramic package. Figure 9 presents the response to a $900 \mathrm{ppm}$ exposure to gaseous hydrogen. The gas was successfully detected, and a recovery time of 50 seconds was achieved. Further testing involved the characterization of the sensor response in different backgrounds. Figure 10 depicts the response to gaseous hydrogen in a $\mathrm{CO}_{2}$ background.

Work is continuing to increase the sensitivity of the nano sensor for hydrogen gas. Among the current activities are:

- Optimization of the 1-D structures of $\mathrm{TiO}_{2}$

- Integration of the $\mathrm{TiO}_{2}$ into a MEMS device Optimization of the MEMS device for enhanced sensitivity, selectivity, and recovery time
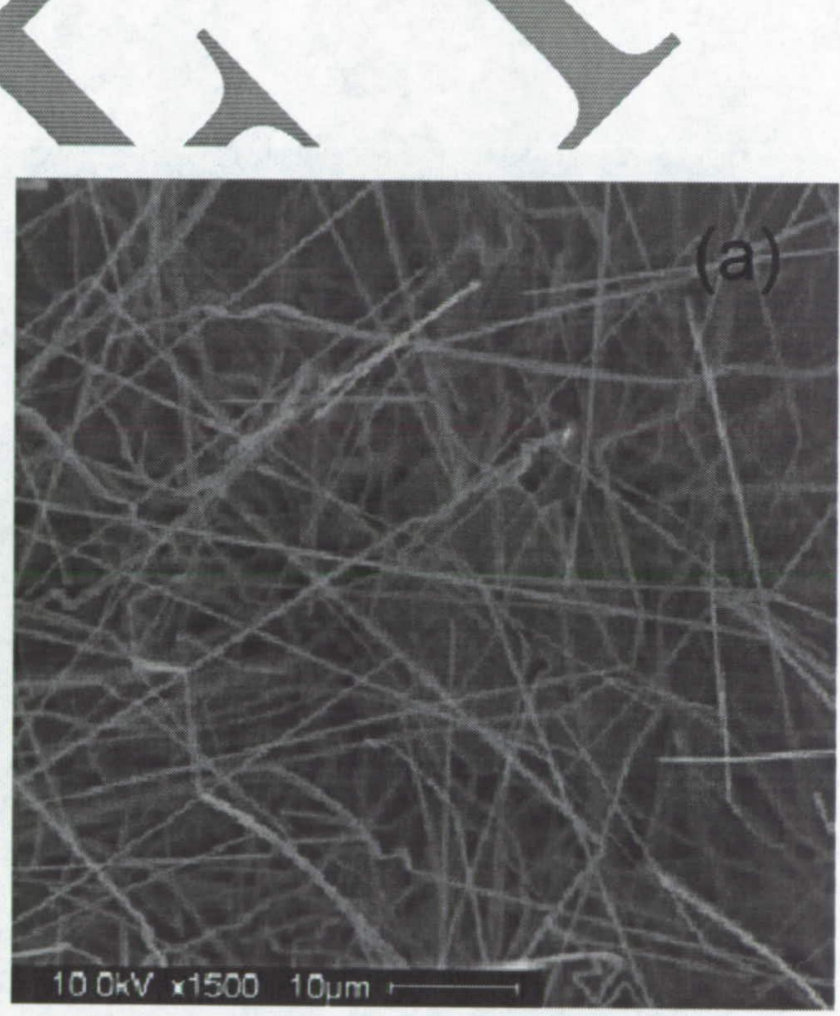

University of Central Florida

Figure 8. SEM images of thermally grown $\mathrm{SnO}_{2}$ wires

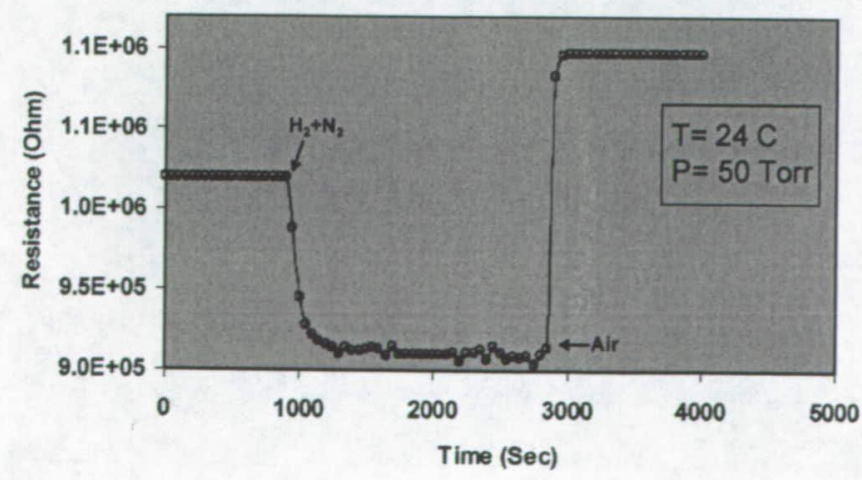


Figure 9. Response of thermally grown tin nanowires to hydrogen gas at room temperature.

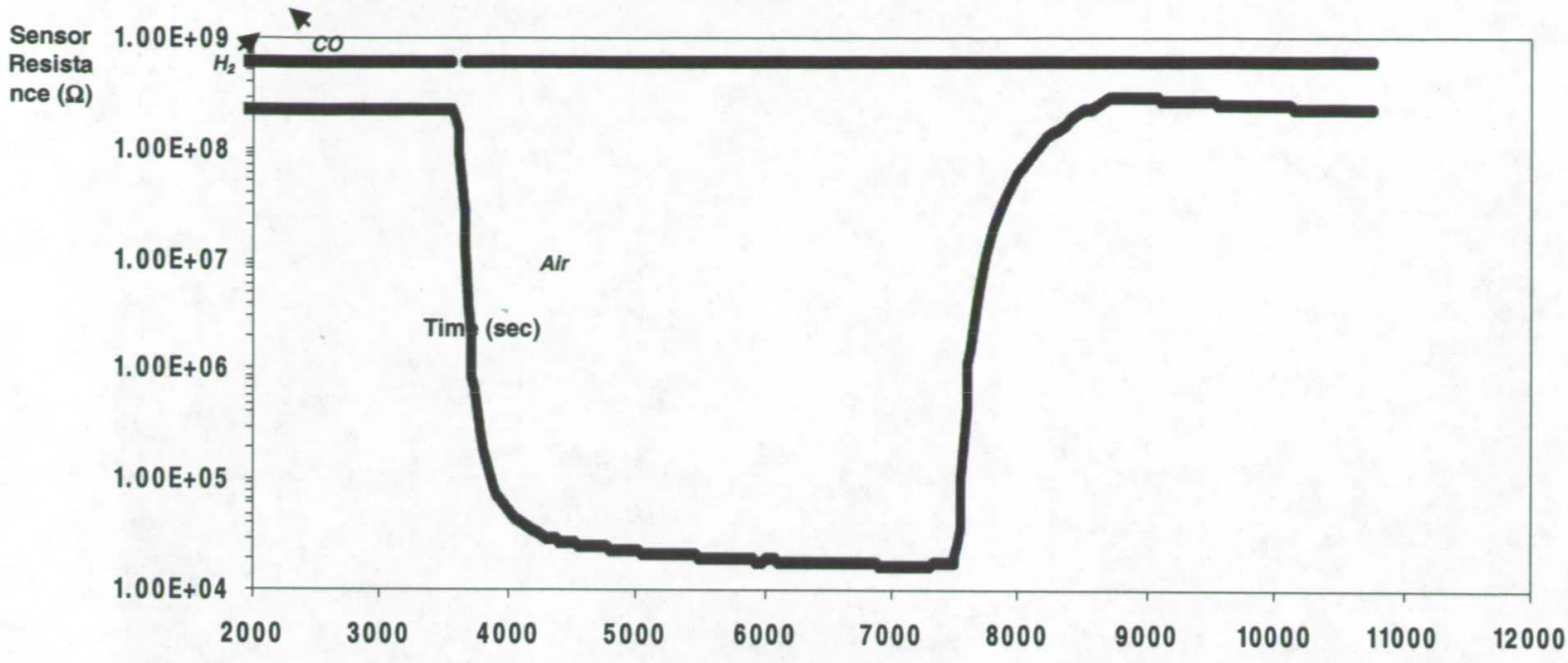

University of Central Florid monoxide background.

\section{SUMMARY}

The development of miniaturized sensors is important for current and future space programs. We have presented some of the development work currently in progress at ASRC and its development partners. Results to date are very encouraging, including both the sensitivity and selectivity obtained with the sensors. Monitoring of the environment, not limited to the detection of gas leaks, will become increasingly important as longer duration missions are planned and executed. A multitude of sensors need to be developed and qualified for space flight under realistic operating conditions, and as such, development activities in the nano sensor field will continue for years to come.

\section{ACKNOWLEDGMENTS}

The author acknowledges the significant contribution of its development partners: Dr. Sudipta Seal and Dr. Hyoung Jin Cho at the University of Central Florida, and Dr. Meyya Meyyappan, Dr. Jing Li, and Dr. Jerry Jin at the Center for Nano Technology Research at NASA Ames Research Center. 